\title{
Vaccination of chickens with live Newcastle disease virus vaccines adjuvanated with Nigella sativa oil
}

\author{
A. S. Hussien and H. M. Madbouly \\ Department of Virology, Faculty of Veterinary Medicine, Beni-Suef University, Beni-Suef Egypt
}

\begin{abstract}
Two hundred, five-week old chickens were divided into six equal groups (30 chicks/group) and vaccinated with different live NDV vaccines in Nigella sativa oil. The vaccinated birds were bled at one-week interval post-vaccination over six weeks and the collected sera were tested by the HI test. Half of each group was challenged 21-days post-vaccination by intramuscular route of inoculation with $2 X_{10}^{5} E^{-1 D} D_{50}$ of the velogenic viscerotropic Newcastle disease virus (VVNDV). Birds were observed for 15 days post-challenge for any clinical signs of Newcastle disease. Deaths within this period were subjected to post mortem examination. The remaining birds in each group were observed after challenge till 42-days post-vaccination.
\end{abstract}

Newcastle disease (ND) is one of the serious and destructive infectious diseases of birds. It is regarded as a major problem facing poultry keeping all-over the world whether in large or small scale. Since the discovery of the disease, great efforts had been made by many workers and investigators to overcome and control it, in order to limit its spread as well as to avoid the damage and drastic economic losses to poultry industry.

The strategy of prevention of ND in chickens depends mainly on vaccination by using either live attenuated or inactivated Newcastle disease virus (NDV) vaccines, which usually protect chickens from the more serious consequences of the disease. Various types of live and inactivated virus ND vaccines are available and their relative merits have been reviewed (Hanson, 1978 and Lancaster, 1981). The potentiality of live-in-oil ND vaccine in chickens of both light and heavy breeds has been reported (Peleg et al., 1985, 1993a, b). In these studies, various lentogenic and mesogenic strains of ND vaccine, when used as live-in-oil vaccines have shown to be superior to aqueous live vaccines or killed adjuvanated vaccines of the same virus strains.

Some studies were planned to avoid the undesirable effect of the mineral oils by replacing them suitable animal, vegetable or synthetic oil as has been described by (Stone, 1997 and Madbouly et al., 2000, 2001 and 2002). Nigella sativa oil was one of these adjuvants that could be used as a replacement for the mineral oil due to its non-specific immunostimulant effect (Basil and Erwa, 1993 and Haq et al., 1995) besides other different desirable effects such as anti-microbial (Hanafy and Hatem, 1991) and anthelmintic effects (Akhtar and Riffat, 1991).

The aim of the present work was to evaluate the effectiveness of mixing the VVNDV or live NDV vaccines (LaSota and Clone 30) with Nigella sativa oil with or without emulsifiers and to compare these different combinations with each other.

\section{Material and methods}

Chicks. One-day-old chickens (local breeds) were purchased from commercial source at Beni-Suef Governorate. On the first day, some chickens were randomly selected and screened for the presence of maternally derived Newcastle disease Virus (NDV) antibodies by using the haemagglutination inhibition test. This test was repeated several times till it showed that all chicks had lost their maternal antibodies and became nearly susceptible to the NDV. All chickens were housed under strict hygienic measures without vaccination against ND till the time of the experiment (five weeks old).

Serum samples. They were collected from the vaccinated and non-vaccinated chickens on detectable periods. Blood samples were individually collected from the wing vein in sterilized Wasserman tubes without anticoagulant and kept in a slope position for clotting at room temperature then for thirty minutes at $37^{\circ} \mathrm{C}$ then 2 hours at $4^{\circ} \mathrm{C}$; followed by centrifugation at $3000 \mathrm{rpm}$ for ten minutes. Supernatant clear serum was then separated and stored individually at $-20^{\circ} \mathrm{C}$ in small sterile Eppindorff tubes till use.

Virus. A virulent strain of NDV (local field isolate) was obtained from the department of 
poultry diseases, Faculty of Veterinary Medicine, Cairo University. It was isolated from a severe outbreak of the Newcastle disease and was identified and characterized as velogenic viscerotropic strain (VVNDV).

NDV vaccines. Both lentogenic LaSota live vaccine; $10^{6.1} \mathrm{EID}_{50} /$ dose, (Intervet International B.V. Boxmeer-Holland serial number 370496) and Clone30; were used in the present study.

Haemagglutination Inhibition test. (Beard and Wilkes, 1973) ( $\beta$ - method) Serial double fold dilutions of anti-NDV serum in physiological saline were prepared using $50 \mu \mathrm{l}$ of $4 \mathrm{HA}$ units of the NDV were added to all wells. The mixture was incubated for half an hour at $37^{\circ} \mathrm{C}$ then $50 \mu 1$ of $1 \%$ suspension of washed chicken R.B.Cs. and the plates were incubated for 15-20 minutes at room temperature before reading the result.

Preparation of the vaccine batch. One part of the aqueous-phase (virus) was mixed with one part of the oil-phase (Nigella sativa oil). The aqueous-phase was added to the oil-phase drop by drop with continuous mixing manually over ten minutes to give homogenized emulsion

Serology. All chickens were bled at weekly intervals post-vaccination for six weeks and the collected sera were tested by the HI test.

Challenge test. Half of chickens each mentioned groups were challenged 21-days postvaccination by the intramuscular inoculation with $2 \times 10^{5} \mathrm{EID}_{50}$ of the VVNDV. The birds were observed for 15-days post-challenge for clinical signs of Newcastle disease. Deaths within this period were recorded and succumbed subjected to post mortem examination to confirm the cause of mortality. The remaining birds in each group were challenged 42-days post-vaccination.

Nigella sativa Oil (whole crude oil). It was prepared from the Nigella sativa seeds (commercial source) by pressing method. The pressed oil was filtered to remove fine seed particles, and then sterilized by filtration through $0.22 \mu \mathrm{m}$ filter (Sartorius-Germany), and kept at $4^{\circ} \mathrm{C}$ till use as adjuvant for vaccines.

Emulsifiers. Sorbitan mono-oleate (SPAN-80): (Ubicham Ltd Code 3045N, Lot 1895) was used for preparing the oil phases of vaccines. Polyoxy ethylene sorbitan (Tween-80); nonionic detergent (Sigma Co., Ltd Code 3265Q, Lot 4540330).

Experimental design. Two hundred, one-day old mixed sex chickens were used in this experiment. They were reared under strict hygienic measures till time of vaccination (five weeks of age) that previously determine by random blood samples, examination using $\mathrm{HI}$ test. The birds were divided into six equal groups, 30 chicks each; and were treated as follow: Group 1: VVNDV + Nigella sativa oil (contain Tween 80 as aqueous phase emulsifier). Group 2:VVNDV + Nigella sativa oil (without Tween 80). Group 3: LaSota vaccine + Nigella sativa oil, Group 4: LaSota vaccine Alone, Group 5: Clone 30 vaccine + Nigella sativa oil, and Group 6: Clone 30 vaccine alone. A control non-vaccinated group was included (group 7).

Each chickens was inoculated $\mathrm{S} / \mathrm{C}$ in the mid-dorsal neck area with a $0.5 \mathrm{ml}$ vaccine dose. Half of each group (3-7) challenged at 21-days post-vaccination while the remaining birds were challenged at 42-days post-vaccination.

\section{Results and Discussion}

Oil adjuvants are readily adopted in many poultry disease antigens and are widely used in water in oil (W/O) emulsion vaccines of poultry. Samberg et al., 1977 stated that the advantage of the system of live-in-oil vaccine might be due to "escape" of infective live virus from the trapping oil environment and initiating infection and replication of the live virus in various tissues and organs, including the respiratory tract.

Data presented in (Table 1) shows clearly that the VVNDV caused severe reactions and high percentages of mortality that reached $100 \%$ in group 1 and $93.33 \%$ in group 2 when given to chickens incorporated in Nigella sativa oil.

Nervous signs, depression, and diarrhea were observed in chickens of group 1 that given VVNDV with Nigella sativa oil contain Tween $80(60 \%)$ at the $5^{\text {th }}$ day post inoculation. Few birds with nervous signs were died while most of the mortalities occurred directly after depression ( $6^{\text {th }}$ day post inoculation). By the $15^{\text {th }}$ day post inoculation only two birds remained alive in this group. On the other hand, the signs of the Newcastle disease in group 2 that treated with VVNDV plus Nigella sativa oil without Tween 80 appeared at the $4^{\text {th }}$ day post inoculation and mortality began at the $5^{\text {th }}$ day post inoculation. The mortality percentage was $100 \%$ in this group.

It was also observed that using of Tween 80 (Aqueous phase emulsifier) and Arlacal A (oil phase emulsifier) with the mixture of virus and oil didn't cause significant effect as detergent on the virus. However, we can't exclude the possibility that the delayed appearance of signs 
Table (1): Daily distribution of deaths in chickens inoculated with VVNDV (Group 1\&2).

\begin{tabular}{cccccccccc}
\hline \multirow{2}{*}{ Group } & Inoculation & $\mathbf{5}^{\text {th }}$ & $\mathbf{6}^{\text {th }}$ & $\mathbf{7}^{\text {th }}$ & $\mathbf{8}^{\text {th }}$ & $\mathbf{9}^{\text {th }}$ & $\mathbf{1 0}^{\text {th }}$ & $\mathbf{1 1}^{\text {th }}$ & $\mathbf{1 2 - 1 5}$ \\
\hline $\mathbf{1}$ & $\begin{array}{c}\text { VVNDV + Nigella sativa } \\
\text { oil (contain Tween) }\end{array}$ & 0 & 4 & 5 & 9 & 8 & 1 & 1 & - \\
$\mathbf{2}$ & $\begin{array}{c}\text { VVNDV + Nigella Sativa } \\
\text { oil (without Tween) }\end{array}$ & 5 & 2 & 10 & 6 & 3 & 4 & - & - \\
\hline
\end{tabular}

Table (2): The mean ND- HI Ab titers in sera of vaccinated chicks.

\begin{tabular}{clcccccc}
\hline \multirow{2}{*}{ Group } & \multirow{2}{*}{ Vaccine } & \multicolumn{5}{c}{ Mean $\log _{\mathbf{2}}$ HI Ab titer per weeks post-vaccination } \\
\cline { 3 - 8 } & & $\mathbf{1}$ & $\mathbf{2}$ & $\mathbf{3}$ & $\mathbf{4}$ & $\mathbf{5}$ & $\mathbf{6}$ \\
\hline $\mathbf{3}$ & LaSota + oil & 2.14 & 3.63 & 4.8 & 3.95 & 3.46 & 2 \\
$\mathbf{4}$ & LaSota alone & 2.16 & 2.86 & 4.45 & 3.46 & 3.25 & 2.8 \\
$\mathbf{5}$ & Clone30 + oil & 1.73 & 3 & 5.4 & 4.93 & 2.9 & 2.4 \\
$\mathbf{6}$ & Clone30 & 2.2 & 3.5 & 5.1 & 4.8 & 3.63 & 2.56 \\
Control & Non-vaccinated & 0 & 0 & 0 & 0 & 0 & 0 \\
\hline
\end{tabular}

Table (3): Protection percentages of vaccinated chickens challenged at 21 and 42 days postvaccination.

\begin{tabular}{clcccc}
\hline \multirow{2}{*}{ Group } & \multirow{2}{*}{ Vaccine } & \multicolumn{2}{c}{ 21 days post-vaccination } & \multicolumn{2}{c}{ 42 days post-vaccination } \\
\cline { 3 - 6 } & & Rate & $\mathbf{\%}$ & Rate & \% \\
\hline $\mathbf{3}$ & LaSota + oil & $11 / 15$ & 73.33 & $4 / 15$ & 26.66 \\
$\mathbf{4}$ & LaSota alone & $10 / 15$ & 66.66 & $6 / 15$ & 40 \\
$\mathbf{5}$ & Clone30 + oil & $12 / 15$ & 80 & $5 / 15$ & 33.33 \\
$\mathbf{6}$ & Clone30 & $11 / 15$ & 73.33 & $6 / 15$ & 40 \\
Control & Non-vaccinated & $0 / 15$ & 0 & $0 / 15$ & 0 \\
\hline
\end{tabular}

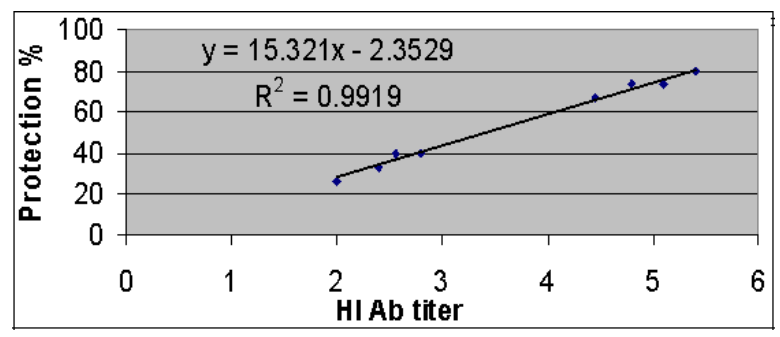

Fig. (1): The correlation between pre-challenge HI antibody titers and post-challenge protection levels in different groups.

and mortalities associated with Tween 80 may be due to the result of a direct interaction of Tween 80 with NDV or the result of direct and independent Tween80 adjuvant effect (Gall, 1967). From these results, it could be concluded that there was no advantage of mixing the VVNDV with oil adjuvants, as there was no significant effect on the virus virulence.

As shown in the results of this work, chicken groups vaccinated with some commercial live NDV vaccines supplemented with Nigella sativa oil elicited different levels of HI antibody titers and mortality percentages. Results of the HI titers and the mortality percentages during the experiment period are presented in (Tables 2 and 3). Generally, the $\mathrm{HI} \mathrm{Ab}$ titers increased gradually from the $2^{\text {nd }}$ week post-vaccination (PV) and reached the peak at the $3^{\text {rd }}$ week PV then decreased gradually with low amounts from the $4^{\text {th }}$ week PV.

On comparing the mean $\log _{2} \mathrm{HI} A b$ titers of chicken group vaccinated with LaSota vaccine alone with that received LaSost plus Nigella sativa oil, it is very clear that the HI Ab titers were nearly similar and there was no great difference. LaSota vaccine alone gave the highest value at $3^{\text {rd }}$ week PV (4.8), while, when mixed with Nigella sativa oil gave a value of (4.45). Concerning the evaluation of the immune response by challenge test, data presented in (Table 3) revealed that when the LaSota vaccine was supplemented with Nigella sativa oil, the

protection level reached $(73.33 \%)$ at 21 days PV and $(26.66 \%)$ at 42 days PV. but, when LaSota vaccine was used alone without the addition of 
oil, the protection level became (66.66\%) and (40\%) at 21 and 42 days PV respectively.

It was observed that chickens vaccinated with Clone 30 adjuvanated with Nigella sativa oil gave higher $\mathrm{HI} \mathrm{Ab}$ titers at $3^{\text {rd }}$ and $4^{\text {th }}$ weeks $\mathrm{PV}$ (5.4 and 4.93) compared to the group given the vaccine only without adjuvant (5.1 and 4.8). On the other hand, these titers were higher with chickens received clone 30 alone at the $2^{\text {nd }}, 5^{\text {th }}$ and $6^{\text {th }}$ weeks PV. Generally, there was no great difference between the groups 5 and 6 as the obtained results were nearly similar. Protection was expected with results of $\mathrm{HI}$ Ab titers induced in this experiment. Results presented in (Table 3) support this finding. For example, the protection percentage at 21 day PV was $(80 \%)$ and $(33.33 \%)$ for chickens vaccinated with Clone30 plus oil and clone 30 alone respectively. The percentage changed to $(73.33 \%)$ and $(40 \%)$ when these chickens were challenged 42 days PV. The protection percentage for the control non-vaccinated was $0 \%$.

Fig. (1) Showed close correlation between the $\mathrm{HI}$ antibody titers of different groups and protection levels $(\mathrm{y}=15.321 \mathrm{x}-2.3529, \mathrm{R} 2=$ $0.9919)$. This result is supported by the fact that protection against NDV challenge is mainly dependent on the presence of sufficient amount of antibody titers, (Box and Furminger, 1975, and Bennejean et al., 1978). In spite of that, the immune response achieved here is still below the level expected. We believed that this is mainly due to the absence of boostering by a second dose of the vaccine.

Finally, there was no advantage of mixing live vaccines with Nigella sativa oils, as there was no significant differences between the groups vaccinated with live vaccine alone and that vaccinated with live-in-oil vaccine at any testing interval post-vaccination relative to $\mathrm{HI}$ $\mathrm{Ab}$ titers or challenge mortality.

\section{References}

Akhtar, M. S. and Riffat, S. (1991): Field trial of Saussurea lapparoots against nematodes and $N$. sativa seeds against cestodes in children. J. Pakistan Med. Assoc., 41: 185-187.

Basil, A. A. and Erwa, H. H. (1993): Effect of $N$. sativa on ingestion ability of mice peritoneal macrophages. Saudi Pharmaceut. J., 1:18.

Beard, C. W. and Wilkes, W. J. (1973): A simple and rapid microtest procedure for determining Newcastle haemagglutination-inhibition antibody titers. Proc. $77^{\text {th }}$ Ann. Mtg. USA An. Health Assoc., pp. 596-600

Bennejean G.; Guittel W. and Picault J. P. (1978): vaccination of one-day old chicks against ND using inactivated oil adjuvant vaccine and/or live vaccine. Avian Pathol., 7: 15-27.

Box P. G. and Furminger I. G. S. (1975): ND antibody levels in chickens after vaccination with oil emulsion adjuvant killed vaccine. Vet. Rec., 96: 108-111.

Gall, D (1967): Observations on the properties of adjuvants. Symp. Ser. Immunobiol. Stand., 6:39.

Hanafy M. S. M. and Hatem M. E. (1991): Studies on the antimicrobial activity of Nigella sativa seed (black cumin). J. Ethnopharmacol., 34: 275-278.

Hanson R. P. (1978): ND. In Hofstad MS $7^{\text {th }}$ ed,. Diseases of Poultry. Ames, Iowa, State Univ. Press, pp., 513-536.

Haq A.; Abdullatif M.; Lobo P. I.; Khabar K. S. A.; Shethik K. V. and Al-Sedairy S. T. (1995): N. sativa: Effect on human phagocytes and polymorphonuclear phagocytic activity. Immunopharmacol., 30:147-155

Lancaster J. E. (1981): Newcastle disease. In E.P.J Gibbs (ed.). Virus dresses of food animals. Disease Monographs. Academic Press, New York, pp., 433-465.

Madbouly H. M., El-Kady M. F., and Tamam S. M. (2000): Preparation of ILT inactivated viral vaccine from locally isolated strain adjuvnated with Nigella sativa oil as adjuvant. Sci.Vet Med. J., III 1: 281-288.

Madbouly H.M., Khashabah, E. M. and Ibrahim, N. M. (2001): Preparation and evaluation of inactivated infectious bursal disease (IBD) virus vaccine from recent Egyptian isolate and adjuvated with Nigella sativa oil. Vet. Med. J., Giza,.49 (4): 553-562.

Madbouly H. M.; Hussein H.A.; Mohammed, F. M.; ElDesawy, O. M.; Saber, S. M. and Shalaby, M. A. (2002): Efficacy of vaccination strategy against lumpy skin disease with the use of new generation of inactivated vaccine. Vet. Med. J. Giza, 50(4):963-975.

Peleg, B. A.; Horenstien, K.; Yadin, H.; Bendheim, U. and Berma, E. (1985): Immunization of chickens with live viral vaccines in oil emulsion. In. J. Samberg (ed.), Proc. Inter. Cong. World Poult. Assoc., Israel, pp.106.

Peleg, B. A.; Samina, I. and Brenner, J. (1993a): Immunization of chickens with live Newcastle disease virus vaccine adjuvanated with oil. Vaccine, 11: 1704-1706.

Peleg, B. A.; Samina, I. and Brenner, J. (1993b): Vaccination of chickens with live fowl pox vaccine in oil. J. Vet. Med. B, 40: 522-524.

Samberg Y.; Horenstein, K.; Cuperstein, E. and Gottfried, R. (1977): Spray vaccination of chickens with an experimental vaccine against Newcastle disease. Avian Pathol., 6: 251-258.

Stone H. D. (1997): Newcastle disease oil emulsion vaccines prepared with animal, vegetable and synthetic oils. Avian Dis., 41:591-597. 